\title{
Thermal systems in process industry as a source for electrical flexibility
}

\author{
Jens Baetens, Brecht Zwaenepoel, Jeroen D. M. De Kooning, Greet Van Eetvelde and Lieven Vandevelde \\ Ghent University (Belgium), Electrical Energy Laboratory (EELAB) \\ Department of Electrical Energy, Metals, Mechanical Constructions \& Systems (EEMMeCS) \\ Contact: J.Baetens@UGent.be
}

\begin{abstract}
The need for balancing services in the European electricity grid is increasing. The reason is twofold: renewable energy sources (RES) are still in up rise, meanwhile the classical power plants - commonly used to provide balancing - are economically pushed out of the market. This results in less available balancing resources but increasing balancing needs as RES are less predictable and controllable. The decline of the balancing volume provided by classical power plants can be counteracted by the concept of Demand Side Response (DSR). Balancing services are shifted towards the electricity consumers instead of traditionally being an electricity producer's responsibility. The concept of DSR is well documented as it was already introduced in 1973 at the time of the first energy (oil) crisis. Now, the DSR concept gains again momentum as electricity markets rapidly change to adopt RES. New insights and incentives are to be investigated as the electrical landscape has changed tremendously since the first introduction of DSR. This paper will focus on the concept of DSR in process industry.
\end{abstract}

Keywords-ancillary services, balancing services, Demand Side Response, electrical flexibility, process industry, thermal systems

Primary conference subject area-Power Systems Operations and Control

\section{INTRODUCTION}

Industry in Europe has a share of $36 \%$ in the total European electricity consumption (2013). A significant part of this consumption can be attributed to very energy-intensive sectors of which process industry is one.

When considering a single plant, a large part of the consumed electricity can usually be addressed to a handful of large consumers on-site. These consumers can be both individual machines and specific subprocesses. These electrical energyintensive sub processes could already contain flexibility, e.g., minimum and maximum operating temperatures, controllable start of batch processes, control of production volumes making use of buffer capacity. Nevertheless this flexibility is not yet actively used to provide balancing services [1].

An advantage of making use of industrial systems to provide electrical flexibility is the already available Supervisory Control And Data Acquisition (SCADA) system or Distributed Control System (DCS) which is commonly in place. Electrical consumers are centrally controlled and the addition of an ancillary service signal to the SCADA system could be sufficient to utilise the electrical flexibility of a system as it is. This is in contrast to residential potential of electrical flexibility where the infrastructure is mostly not available and can be a costly operation to install.

A possible drawback of implementing DSR in industry is the safety aspect. When considering the use of certain processes or machines to provide electrical flexibility, the safety constraints must be maintained at all times.

When looking at process industry from a DSR viewpoint, processes can be divided into three main groups: core processes (e.g. extrusion, oxidation reaction and electrolysis), utilities (e.g. waste water treatment, cooling and heating) and own generation of electricity (e.g. steam turbine, CHP unit and diesel backup generator). All three groups could possibly provide electrical flexibility. In this paper we will focus on the utilities part of the process industry, more specifically the thermal processes of electric heating and the electric consumption in cooling.

Process industry, and industry in general, often requires significant amounts of heating and cooling. These systems can be considered as Thermal Energy Storage (TES) units, although their main purpose is to efficiently heat and cool and not to function as storage or buffer. When considering the thermal inertia of such systems, large thermal time constants could be coupled to shorter electrical time constants of ancillary services. This way a thermal system could provide balancing services to the electricity grid while maintaining the safe operation boundaries of the process.

In the total energy mix used to provide heating in industry, electric energy plays a rather small roll. Combined Heat and Power (CHP) units are used to foresee in both heating and generation of electricity. Also common is the use of heat integration to transfer excess heat from one process to another by using heat exchangers. Nevertheless, electric heating is present in certain specific cases such as tracing wires and electric back-up boilers and often take up a significant part of the total electricity consumption.

This paper will focus on the use of existing heating and cooling systems in process industry with significant installed power and electrical consumption to provide balancing services. Methods will be proposed on how to implement specific service types of currently available balancing services to industry sized systems, in which also the safety aspect will be taken into account.

In $\S \Pi$ the balancing markets with their three main products will be described, as defined by ENTSO-E and applied to the Belgian transmission system. Next, in $\S$ III the process of electric heat tracing is explained and a possible electrical flexibility case has been worked out. $\S$ IV]deals with an electric chiller and defines thermal time constants, based on an industry sized unit. This process is then coupled to an ancillary service product so to use its available electrical flexibility.

\section{BALANCING MARKETS}

The synchronous grid of Continental Europe, also known as Continental Synchronous Area is the largest synchronous electrical grid in terms of connected power. The grid is operated by Transmission System Operators (TSOs) which are grouped in the European Network of Transmission System Operators for Electricity (ENTSO-E). ENTSO-E provides an Operational Reserves framework to TSOs on implementing ancillary services to efficiently balance the electricity grid [2], [3]. 
As the framework is translated to ancillary service products by each TSO - and is thus mostly country dependent - a wide variety of services exists. In this section the three main ancillary services as defined by the ENTSO-E will be generally discussed as well as their implementation by the Belgian TSO (Elia), with specific attention to the timestamps.

\section{A. Frequency containment reserves}

Frequency containment reserves (FCR), formerly known as R1 or primary reserves are being used to stabilise the grid frequency within a short time-frame of seconds. Frequency deviations are counteracted by up- or downward regulation respectively for deviations below and above nominal frequency. A continuous adaptation of the power setpoint based on the grid frequency needs to be implemented.

Elia offers four different FCR service types, presented here with their formula to determine the required power in function of the grid frequency and visualised in Fig. 1]

\section{- Symmetric FCR $200 \mathrm{mHz}$}

$$
\begin{gathered}
49.8 \mathrm{H} z \leq f \leq 50.2 \mathrm{H} z: \\
P_{\text {req }, 200 \mathrm{mHz}}=-\lambda_{0} P_{\mathrm{b}} \Delta f \\
f<49.8 \mathrm{H} z: \\
P_{\text {req, } 200 \mathrm{mHz}}=P_{\mathrm{b}} \\
f>50.2 \mathrm{H} z: \\
P_{\text {req }, 200 \mathrm{mHz}}=-P_{\mathrm{b}}
\end{gathered}
$$

\section{- Symmetric FCR $100 \mathrm{mHz}$}

$$
\begin{gathered}
49.9 \mathrm{~Hz} \leq f \leq 50.1 \mathrm{~Hz}: \\
P_{\text {req, } 100 \mathrm{mHz}}=-2 \lambda_{0} P_{\mathrm{b}} \Delta f \\
f<49.9 \mathrm{~Hz}: \\
P_{\text {req }, 100 \mathrm{mHz}}=P_{\mathrm{b}} \\
f>50.1 \mathrm{H} z: \\
P_{\text {req }, 100 \mathrm{mHz}}=-P_{\mathrm{b}}
\end{gathered}
$$

\section{- Asymmetric Down FCR}

$$
\begin{gathered}
50.1 \mathrm{H} z \leq f \leq 50.2 \mathrm{H} z: \\
P_{\text {req,down }}=-2 \lambda_{0} P_{\mathrm{b}}(\Delta f-0.1 \mathrm{H} z) \\
f>50.2 \mathrm{H} z: \\
P_{\text {req, down }}=-P_{\mathrm{b}}
\end{gathered}
$$

\section{- Asymmetric Up FCR}

$$
\begin{gathered}
49.8 \mathrm{H} z \leq f \leq 49.9 \mathrm{H} z: \\
P_{\text {req,up }}=-2 \lambda_{0} P_{\mathrm{b}}(\Delta f+0.1 \mathrm{H} z) \\
f<49,8 \mathrm{H} z: \\
P_{\text {req,up }}=P_{\mathrm{b}}
\end{gathered}
$$

$P_{\text {req }}$ is the required power to supply for the each specific product, $P_{\mathrm{b}}$ is the contracted power, $\Delta f$ is the grid frequency deviation and $\lambda_{0}$ is the power/frequency characteristic of the ENTSO-E, currently equal to 5 .

The activation of the FCR power needs to start at latest 2 seconds after the start of a frequency deviation and needs to rise linearly to $50 \%$ after a maximum of 15 seconds and to $100 \%$ after a maximum of 30 seconds.

Elia can perform a test to the FCR providing system in which a step-by-step frequency deviation is simulated. The exact parameters of this test are specific for each of the service types, but requires that the full contracted capacity $P_{\text {req }}$ stays activated for a certain amount of time [4], [5].

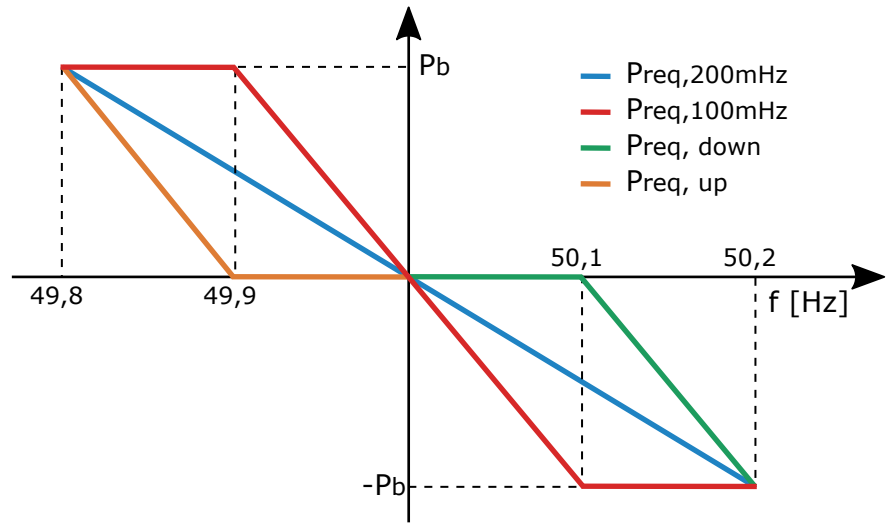

Fig. 1. Requested power in function of the grid frequency for all Elia FCR service types. Based on [4].

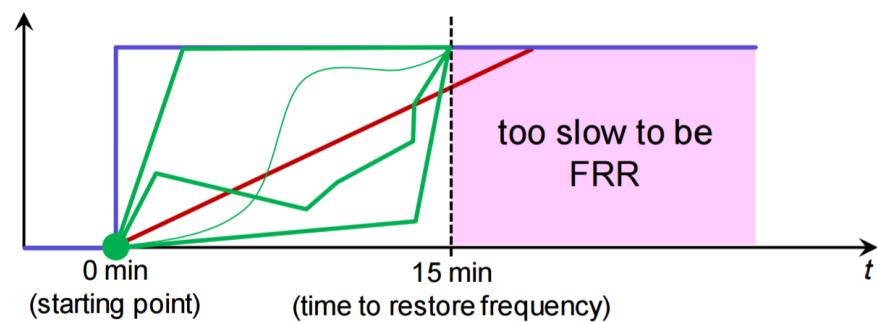

Fig. 2. TTRF as separation point between FRR and RR. Blue line is the TSO request, green lines are allowed responses for FRR and red line is not allowed for FRR [8].

\section{B. Frequency restoration reserves}

Frequency restoration reserves (FRR), formerly known as R2 or secondary reserves are used to restore the system frequency. Both automatic (aFFR) and manual (mFFR) reserves exist in the ENTSO-E framework. aFFR is activated upwards or downwards automatically and continuously by signals sent by the TSO's central Load Frequency Controller (LFC), typically every 4 to $10 \mathrm{~s}$. The mFFR is discretely and ad-hoc decision based with typically a larger Full Activation Time (FAT) [6].

Currently Elia contracts only FRR reserves to generators and not to electrical loads. Two services can be contracted:

- Upward Secondary Control

- Downward Secondary Control

Both services have a FAT of 7.5 minutes. [7]

\section{Replacement reserves}

Replacement reserves (RR), formerly known as R3 or tertiary reserves are the third and lastly activated reserves. All electrical flexibility products with FATs exceeding the Time To Restore Frequency (TTRF), are considered RR. With a TTRF in Europe of 15 minutes, Fig. 2 shows allowed and nonallowed response curves for FRR providing systems. Systems with non-allowed (too slow) FRR providing curves could be used to provide RR. Note that also reserves with activation times less than TTRF can be contracted as RR.

Elia contracts three RR products, which are catalogued as reserved power and are listed below.

- R3 Standard: Activation up to 8 hours per day, for an unlimited number of activation times. FAT of 15 minutes.

- R3 Flex: Activation up to 2 hours per day, with a maximum of 8 activations per delivery period. FAT of 15 minutes. 


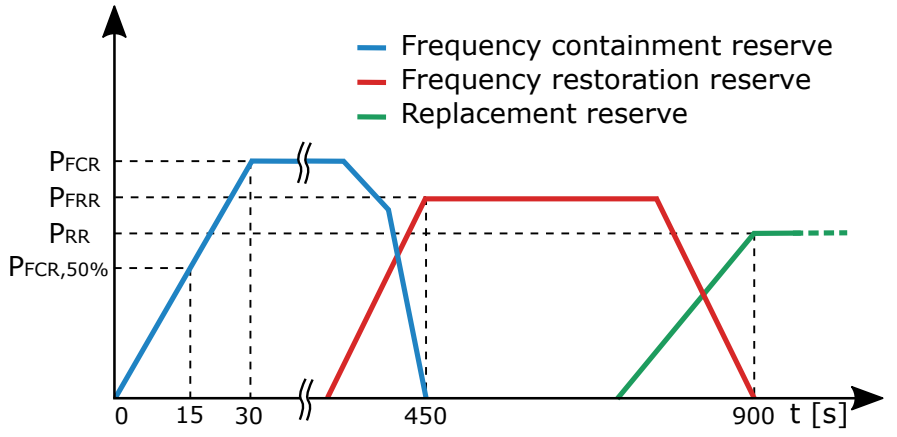

Fig. 3. Cascade of activated balancing reserves in case of a grid disturbance. Based on [2]

- ICH: Interruptible loads with a FAT of 3 minutes and a maximum duration of 4 hours (A4) or a maximum duration of 8 hours (A8).

Other possible RR service that can be provided to Elia is the "Tertiary Control Non-Reserved Power" which makes use of a day-ahead bidding market per quarter hour. Bids can be placed by providing the specific time of availability, available power (up- or downwards regulation) and a fixed price in euro/MW/h.

Fig. 3 gives a schematic overview of the ancillary services with sequence of FCR, FRR and RR as defined by the ENTSOE.

In $\S$ III and $\S$ IV two examples of heating and cooling systems frequently used in process industry will be discussed and will be coupled to a specific balancing service as discussed in $\oint$ II

\section{HEATING IN INDUSTRY: TRACING}

\section{A. Working principle}

In tracing - electric resistive wire heating - the two most common applications in industry are freeze protection and temperature maintenance. Freeze protection tracing is used to prevent pipes or vessels from freezing in winter and are thus a seasonal application. Freeze protection only requires heat at low temperatures, a low power $(10 \mathrm{~W} / \mathrm{m}$ to $30 \mathrm{~W} / \mathrm{m})$ tracing wire can be used.

Temperature maintenance is a continuous process throughout the year that keeps products from solidifying or condensing when being transported through pipes or vessels. When operating such a continuous process to be maintained at super ambient temperature, a constant loss of heat to the surroundings is present. The amount of heat that needs to be added to compensate for this heat loss is dependent on insulation, size of pipes, ambient temperature, product flow and others. Tracing wires with a rated power of $25 \mathrm{~W} / \mathrm{m}$ to $160 \mathrm{~W} / \mathrm{m}$ can be used [9].

The total installed power of tracing wire on a large scale industrial site can be situated between $100 \mathrm{~kW}$ up to a few MWs, with an installed length of several kilometres.

Different technologies in electric tracing exist, of which constant power and self-regulating being the most established ones in industry. Both cables are easy to install as they can be powered with a standard voltage (230 Vac or 400Vac) and can be cut to the necessary length.

With constant power cables, resistors between both conductors are used to generate heat, independent from the ambient or pipe temperature. Control is usually done with a thermostat, switching the power to the cable based on temperature feedback from the pipes with a certain hysteresis. For safety reasons thermostat setpoints are usually set higher

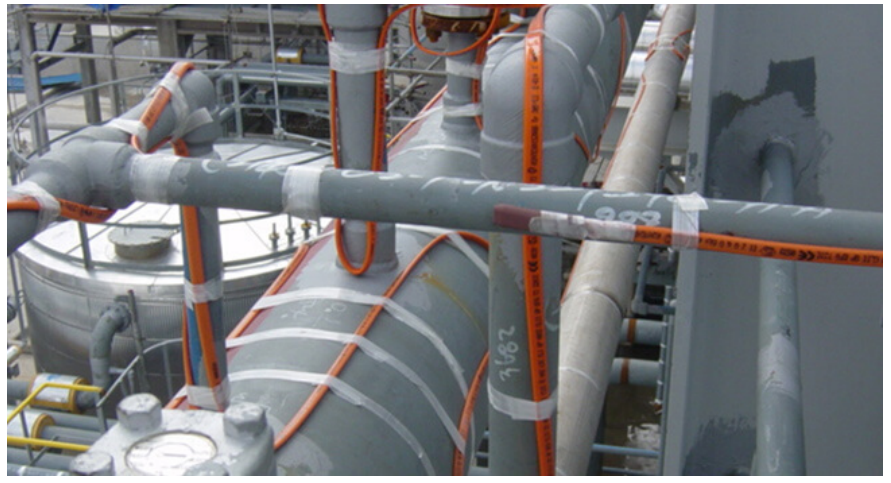

Fig. 4. Industry example of heat tracing placed on pipes [10].

than necessary for the process, this to prevent cold spots from falling below the minimum required temperature.

Self-regulating cables are made from temperature sensitive material, the resistance of which is directly proportional with temperature. This type of wires have a fixed temperature setpoint and cannot be externally controlled. Because of the self-regulation, the total amount of power consumed by the system is hard to define or predict in different process states. The main advantage of this type of tracing wire is the energyefficiency; the cable keeps the whole pipe to a homogeneous fixed temperature and only heats where necessary. Disadvantage is the disability to set different temperatures and the disability to externally control the power to the cable.

\section{B. Balancing service potential}

Older tracing systems frequently make use of a thermostat to control the power. This type of control creates on/off power cycles with a frequency of 10ths of seconds to several minutes, mainly depending on the thermal time constant of the system and the hysteresis of the thermostat. The duty cycle of the signal is dependent on the heat loss and heat addition from and to the system and, averaged over time, can be seen as the power load factor (LF) of the total system. E.g. a LF of 0.6 will correspond with an average duty cycle of $60 \%$. Assuming that the LF is not 1, both upwards and downward regulation of the total consumed power is possible [11].

More modern tracing power controllers comprise of a analogue or digital system driving a Continuously Variable Power Source (CVPS) such as an AC chopper, a rectifier with a DC chopper or fast switching solid state relay (SSR). A PID control strategy is often implemented in the system with temperature feedback from the pipes or vessels. As the power can be controlled continuously, a more narrow temperature control range can be used. Considering the physical properties of such a modern system - a fast reacting PID controller coupled to a continuously variable power source supplying a resistive load - this system could be able to provide FCR service [12].

When contracting a specific FCR service type, the corresponding requested power curves need to be followed as explained in $\S$ II-A and Fig. 11. To do so, a second power control signal is introduced to the existing control loop as well as an Intelligent Power (IP) controller (Fig. 5). This second power control signal implements the requested power curves based on the grid frequency. The IP controller combines both power control signals $\left(P_{\mathrm{PID}}\right.$ and $\left.P_{\mathrm{FCR}}\right)$ and controls the CVPS and thus the power supplied to the tracing system.

With two power control signals, instability or counter control activity could arise, e.g. while the $P_{\mathrm{FCR}}$ requests an upward regulation (less power consumption) of the tracing power 
because of a grid frequency dip, $P_{\text {PID }}$ requests a downward regulation (more power consumption) due to a drop in temperature. In such cases the IP controller will decide the actual power that needs to be supplied to the tracing system, based on level of importance of both signals. Following method is proposed to implement in the IP controller, based on a set temperature range in which the tracing wire should operate in terms of process safety.

$$
\begin{gathered}
T_{\mathrm{min}}<T_{\mathrm{safe}}<T_{\mathrm{max}} \text { and } P_{\mathrm{FCR}}(t)>P_{\mathrm{PID}}(t) \text { and } \\
\left(\frac{\delta P_{\mathrm{PID}}(t)}{\delta t}>0 ; P_{\mathrm{FCR}}(t)>0 \text { or } \frac{\delta P_{\mathrm{PID}}(t)}{\delta t}<0 ; P_{\mathrm{FCR}}(t)<0\right): \\
P_{\mathrm{t}}(t)=P_{\mathrm{FCR}}(t) \\
T_{\mathrm{min}}<T_{\mathrm{safe}}<T_{\max } \text { and } P_{\mathrm{PID}}(t)>P_{\mathrm{FCR}}(t) \text { and } \\
\left(\frac{\delta P_{\mathrm{PID}}(t)}{\delta t}>0 ; P_{\mathrm{FCR}}(t)>0 \text { or } \frac{\delta P_{\mathrm{PID}}(t)}{\delta t}<0 ; P_{\mathrm{FCR}}(t)<0\right): \\
P_{\mathrm{t}}(t)=P_{\mathrm{PID}}(t) \\
T_{\mathrm{min}}<T_{\mathrm{safe}}<T_{\mathrm{max}} \text { and }\left(\frac{\delta P_{\mathrm{PID}}(t)}{\delta t}>0 ; P_{\mathrm{FCR}}(t)<0\right. \text { or } \\
\left.\frac{\delta P_{\mathrm{PID}}(t)}{\delta t}<0 ; P_{\mathrm{FCR}}(t)>0\right): \\
P_{\mathrm{t}}(t)=P_{\mathrm{FCR}}(t) \\
T_{\min }>T_{\mathrm{safe}}>T_{\max }: \\
P_{\mathrm{t}}(t)=P_{\mathrm{PID}}(t)
\end{gathered}
$$

with

$$
\frac{\delta P_{\mathrm{PID}}(t)}{\delta t}=P_{\mathrm{PID}}(t-1)-P_{\mathrm{PID}}(t) .
$$

Applying this control strategy, the providing of the FCR service has priority on the PID temperature control loop, as long as the system operates within the set temperature range.

In case of opposing power signals $(3$ this results in periods when the temperature will deviate more from its setpoint due to providing the balancing service. This will result in an increasing $P_{\mathrm{PID}}(t)$, which will be set if conditions (2) are met. The $P_{\mathrm{t}}(t)$ will then exceed the necessary $P_{\mathrm{FCR}}(t)$ to contractually provide, so to increase or decrease temperature. As this is beneficial for the electricity grid, no penalty will be paid to the TSO.

In case the safety temperature range is not met, $P_{\mathrm{PID}}(t)$ will be set, in order to increase or decrease temperature. When this happens, the contractually agreed $P_{\mathrm{FCR}}(t)$ is not provided and a penalty will need to be paid to the TSO. An optimum should be found between the total contracted FCR power in relation to average consumed power and the process safety temperature range so to minimise the exceeding of the temperature ranges and the penalty payments.

Such a control loop as described above controls only a certain part of the total installed tracing wire. Combining several of these systems could be beneficial, e.g. as one system fails to deliver the necessary $P_{\mathrm{FCR} \text {,system } 1}(t)$, another system might be able to provide the shortcoming $P_{\mathrm{FCR} \text {,system1 } 1}(t)+$ $P_{\mathrm{FCR}, \text { system2 }}(t)$ and thus the total contracted $P_{\mathrm{FCR}}(t)$ could be met. The combination of several of these systems and their ability to provide FCR should be further modelled prior to implementing this control strategy.

\section{COOLING IN INDUSTRY: CHILLER}

\section{A. Working principle}

The working principle of a chiller is to extract heat from a cold reservoir, to transfer this heat to a hot reservoir and this by

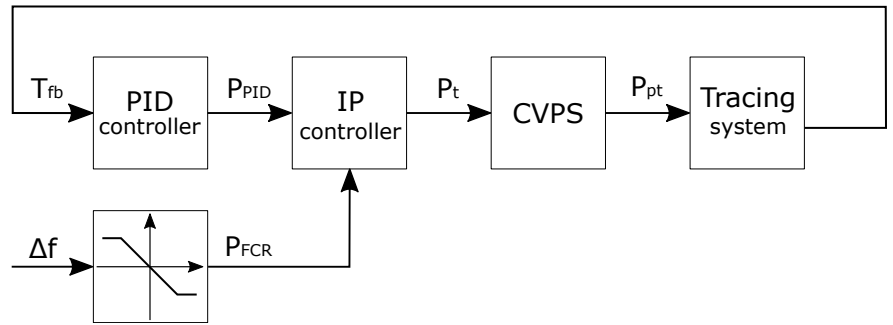

Fig. 5. Schematic representation of a tracing system and its control strategy with the proposed adaptations to implement FCR service.

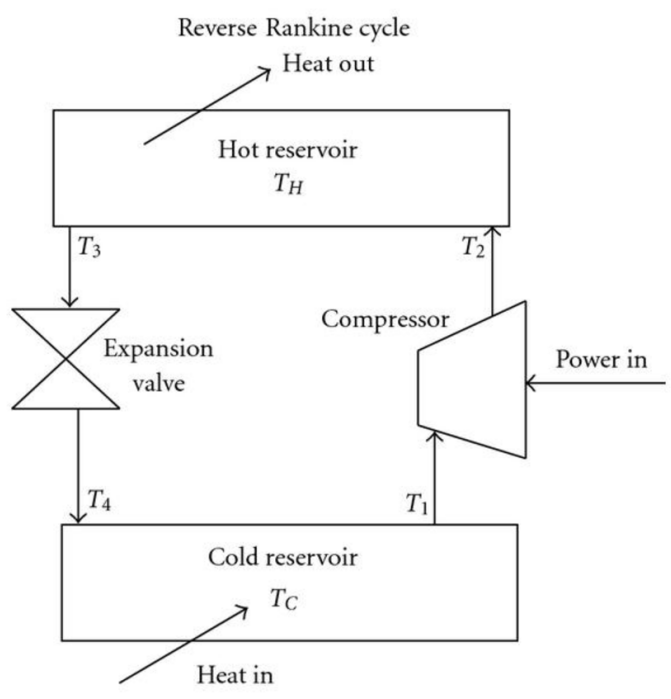

Fig. 6. Schematic representation of a reverse Rankine cycle [13].

applying power to the system. The Coefficient Of Performance (COP) indicates how much heat is extracted in relation to the applied electric power. Different technologies in chillers exist of which the "reverse-Rankine" cycle also known as "vapourcompression" is the most widely used in industry. The electric power added to the system is in form of powering a motor to drive a compressor, so to transport the heat to the hot reservoir.

The main important factors to consider in an industrial chiller system, in terms of electrical flexibility, are the size of the cold reservoir, the installed power and the control system of the compressor. The size of the compressor can vary from several $\mathrm{kWs}$ to multiple MWs, depending on the magnitude of heat that needs to be extracted. Where originally these compressors were switched Direct On Line (DOL), more modern systems are equipped with a Variable Speed Drive (VSD), so to efficiently control the amount of heat extracted from the cold reservoir and reduce the compressor switching. The cold reservoir creates a thermal buffer between the chilled water producer, being the chiller and the chilled water consumer, being the process.

\section{B. Balancing service potential}

To define a magnitude of the thermal time constant of an industrial scaled chiller, some rudimental calculations can be made. Considering an electric DOL switched chiller consisting of a 1MW compressor with an efficiency of $90 \%$ and a COP of 4 , the cooling power $Q_{\mathrm{c}}$ can be calculated as follows.

$$
\dot{Q}_{\mathrm{c}}=\eta \cdot C O P \cdot \dot{Q}_{\text {input }, \mathrm{e}}=3.6 \mathrm{MW}
$$

The heat produced - and to be evacuated by using the chilled water - by the process, $Q_{\mathrm{p}}$, is presumed to be constant at 
2.6MW. We consider a case with a $50 \mathrm{~m}^{3}$ cold water reservoir and a hysteresis of $2^{\circ} \mathrm{C}$ (small buffer case), and a case with a $200 \mathrm{~m}^{3}$ cold water reservoir and a hysteresis of $6^{\circ} \mathrm{C}$ (large buffer case). Losses are neglected and the only variance in temperature is supposed to be through heat exchange with the chiller or the process.

$$
\frac{\delta T}{\delta t}=\frac{\dot{Q}_{\mathrm{p}}-\dot{Q}_{\mathrm{c}}}{C_{\mathrm{w}} \cdot m_{\mathrm{r}}}
$$

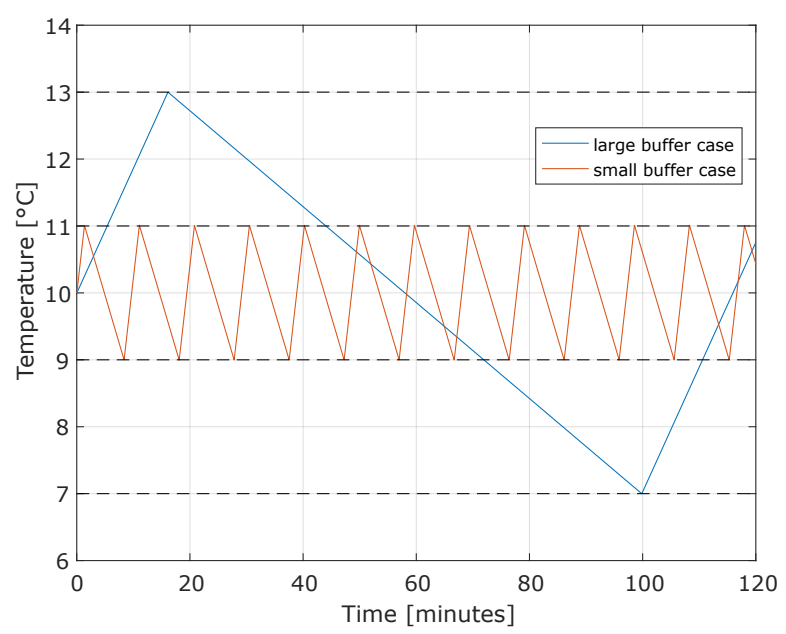

Fig. 7. Cold reservoir thermal profiles of a large and small electric chiller buffer case.

Fig. 7 shows the temperature curve for both cases. While the small buffer case creates cycles with a timespan of 10 minutes, the large buffer case creates cycles with a timespan of 114 minutes, both with an average LF of 0.72 .

The enlargement of the cold reservoir buffer volume increases the cycle time which in turn increases the potential to participate in FRR or RR balancing services.

We will now consider the possibility of using this large buffer chiller case to provide RR "Tertiary Control NonReserved Power" service as described in $\S$ III-C. With an average LF of 0.72 room is available to shift the active compressor time. The idea is to schedule the active time of the compressor to predefined moments so to place bids for an upwards (load reduction) RR service on the day-ahead bidding market. E.g., a period of 15 minutes of scheduled active compressor time will be considered for each hour, thus a $25 \%$ of total time, and will be scheduled from $\mathrm{xx}: 15 \mathrm{~h}$ to $\mathrm{xx}: 30 \mathrm{~h}$.

Despite implementing this RR balancing service, the minimum and maximum temperatures as originally set in the temperature hysteresis need to be preserved at all times. To do so, an extra temperature range $T_{\mathrm{pd}}$ to $T_{\mathrm{pr}}$ is defined. At the start of each scheduled period, the temperature $T_{\text {start }}$ needs to be within this range.

$$
\begin{gathered}
T_{\mathrm{pr}}>T_{\mathrm{start}}>T_{\mathrm{pd}} \\
T_{\mathrm{pr}}=T_{\max }-\frac{\delta T_{\mathrm{r}}}{\delta t} t_{\mathrm{s}} \\
T_{\mathrm{pd}}=T_{\min }-\frac{\delta T_{\mathrm{d}}}{\delta t} t_{\mathrm{s}}
\end{gathered}
$$

with $\frac{\delta T_{\mathrm{r}}}{\delta t} t_{s}$ the possible rise in temperature during the scheduled period and $\frac{\delta T_{\mathrm{d}}}{\delta t} t_{s}$ the possible decline in temperature during the scheduled period.

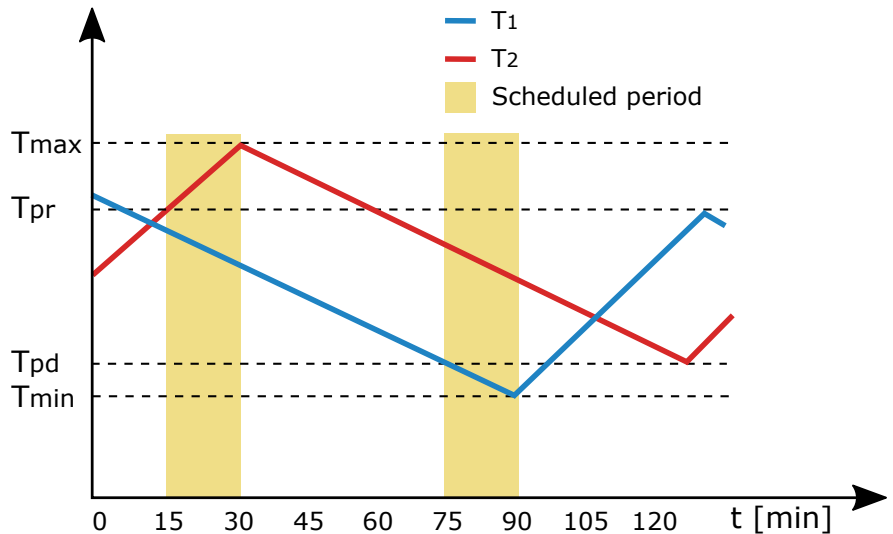

Fig. 8. Scheduled time frames with two extreme conditions, reaching the minimum and maximum temperatures.

Fig. 8 gives a visual presentation of the scheduled time frames and two possible temperature curves, both reaching an extreme condition. A first curve $\left(\mathrm{T}_{1}\right)$ is shown when at the start of the scheduled period $T_{\mathrm{start}}=T_{\mathrm{pd}}$ and no activation takes place so the temperature will decline to $T_{\min }$. Curve $T_{2}$ shows the opposite extreme; when at the start of the scheduled period $T_{\text {start }}=T_{\mathrm{pr}}$ and an activation takes place, the temperature will rise to $T_{\max }$. In both cases the temperature extremes are reached, but not exceeded. As soon as the scheduled period ends, the compressor is freed from providing the RR service and can adapt the temperature again by respectively switching the compressor off or on.

Considering this simplified example, RR service providing by an electric chiller is possible. Nevertheless, more detailed modelling with dynamic heat loads and taking into account possible losses should be carried out in advance to implementing a real case.

Considering the safety aspect, one more remark can be made. If a non-constant or non predictable process heat load $Q_{\mathrm{p}}$ is considered, the temperature extremes could be exceeded by sudden temperature swings. A possible measure could be respectively lowering and increasing $T_{\mathrm{pr}}$ and $T_{\mathrm{pd}}$ creating a buffer, avoiding the temperatures to reach its extremes. A possible drawback of this implementation is the more narrow temperature range for $T_{\text {start }}$ which could lead to more compressor start/stops. An optimum should be found, based on the predictability of heat load, compressor start/stops (and the coherent maintenance cost) and the temperature extremes importance to the safe working of the process.

\section{CONCLUSions}

In this paper, the idea of using existing thermal systems in process industry to provide balancing services is investigated. This approach is led by a fast changing electricity and balancing market as it is adapting to increase RES integration. Future grid balancing markets could comprise a large amount of small ancillary service providers, this in comparison with today's market where larger fossil fuel power plants take the balancing role. As a large electricity consumer, process industry could take a lead role in this. By using their existing, non-core process thermal systems, balancing could be provided without compromising or even influencing the core process.

An overview is given of the balancing market products as defined by the ENTSO-E and application to the Belgian electricity grid operated by Elia. Two commonly available thermal systems - tracing and chilling - in process industry are discussed. The working principle is explained and a magnitude 
of thermal time constant is proposed. A control strategy is defined to implement a specific type of balancing service.

The first case couples electric heat tracing to frequency containment reserves by adding a second power control signal to an existing temperature feedback PID system. A strategy is proposed to control the power to the tracing system based on level of importance of both power control signals. The opportunity of combining several of these systems is also discussed.

A second case studies the possibility of an electric chiller to provide restoration reserve by using the "Tertiary Control NonReserved Power" service. Using the temperature hysteresis of the chiller in combination with the thermal inertia of the cold reservoir, scheduling of the active compressor time could create a system able to provide this service.

Both cases give a general idea on how to implement ancillary services on existing thermal systems focussed on technical possibilities. Both economic and legal aspects should be considered before implementing such cases, e.g. taking into account the necessary tender block sizes required by the TSO or the increased maintenance cost by adapting the existing system etc., but this is out of the scope of this paper.

\section{REFERENCES}

[1] Davide Fabozzi; Nina F. Thornhill; Bikash C. Pal, "Frequency restoration reserve control scheme with participation of industrial loads," in IEEE PowerTech, vol. 5211, Grenoble, France, June 2013.
[2] Continental Europe Operation Handbook - Policy 1: Load-Frequency Control and Performance, 2nd ed., ENTSO-E, Brussels, Belgium, April 2009 .

[3] Operational Reserve Ad Hoc Team Report, 6th ed., ENTSO-E, Brussels, Belgium, June 2012, final version as of 23/05/2012.

[4] General Framework for Frequency Containment Reserve Service by Non-CIPU resources, Elia, Brussels, Belgium, May 2017.

[5] General Framework for Frequency Containment Reserve Service by CIPU resources, Elia, Brussels, Belgium, May 2017.

[6] Impact of Merit Order activation of automatic Frequency Restauration Reserves and harmonised Full Activation Times, 1st ed., Elia, Brussels, Belgium, February 2016, on behalf of ENTSO-E.

[7] General framework for Secondary Control Service by Generators, Elia, Brussels, Belgium, May 2017.

[8] Proposal for mFRR and RR Standard Products - supporting document, ENTSO-E, Brussels, Belgium, March 2016.

[9] Engineering Specification for Electrical Heat-Tracing Systems, Pj333 ed., Chromalox, April 2015.

[10] "Heat tracing," https://petrocheminc.com/services/ other-industrial-services.html accessed: 2017-05-13.

[11] Peter R. Baen, "The value of controls and monitoring for electrical heating tracing," in IEEE Transactions on Industry Applications, vol. 31, no. 2, March 1995, pp. 429-437.

[12] IntelliTrace ITC1 and ITC2 Digital Heat Trace Controller, Chromalox, http://www.chromalox.com/-/media/files/catalog/resources/en-us/ mod-itc1_itc2.pdf

[13] Mahmoud Huleihil; Bjarne Andresen, "Generalized performance characteristics of refrigeration and heat pump systems," vol. 11, 2010. 